\title{
MATE: MPLS Adaptive Traffic Engineering
}

\author{
Anwar Elwalid \\ Bell Labs \\ Lucent Technologies \\ Murray Hill, NJ
}

\author{
Cheng Jin \\ EECS Dept \\ Univ. of Michigan \\ Ann Arbor, MI
}

\author{
Steven Low \\ EE Dept \\ Caltech \\ Pasadena, CA
}

\author{
Indra Widjaja \\ Fujitsu Network Communications \\ Pearl River, NY
}

\begin{abstract}
Destination-based forwarding in traditional IP routers has not been able to take full advantage of multiple paths that frequently exist in Internet Service Provider Networks. As a result, the networks may not operate efficiently, especially when the traffic patterns are dynamic. This paper describes a multipath adaptive traffic engineering mechanism, called MATE, which is targeted for switched networks such as MultiProtocol Label Switching (MPLS) networks. The main goal of MATE is to avoid network congestion by adaptively balancing the load among multiple paths based on measurement and analysis of path congestion. MATE adopts a minimalist approach in that intermediate nodes are not required to perform traffic engineering or measurements besides normal packet forwarding. Moreover, MATE does not impose any particular scheduling, buffer management, or a priori traffic characterization on the nodes. This paper presents an analytical model, derives a class of MATE algorithms, and proves their convergence. Several practical design techniques to implement MATE are described. Simulation results are provided to illustrate the efficacy of MATE under various network scenarios.
\end{abstract}

\section{INTRODUCTION}

\section{A. Motivation}

Internet Service Providers (ISPs) are facing the challenge of designing their networks to satisfy customers' demands for fast, reliable, and differentiated services. Internet traffic engineering is emerging as a key tool for achieving these goals in a cost-effective manner. According to the IETF, Internet traffic engineering is broadly defined as that aspect of network engineering dealing with the issue of performance evaluation and performance optimization of operational IP networks [2]. More specifically, traffic engineering often deals with effective mapping of traffic demands onto the network topology, and adaptively reconfiguring the mapping to changing network conditions. It is worth noting that traffic engineering is more general than QoS routing in the sense that traffic engineering typically aims at maximizing operational network efficiency while meeting certain constraints, whereas the main objective in QoS routing is to meet certain QoS constraints for a given sourcedestination traffic flow.

The emergence of MultiProtocol Label Switching (MPLS) with its efficient support of explicit routing provides basic mechanisms for facilitating traffic engineering [7], [14]. Explicit routing allows a particular packet stream to follow a pre-determined path rather than a path computed by hop-by-hop destinationbased routing such as OSPF or IS-IS. With destination-based routing as in traditional IP network, explicit routing can only be provided by attaching to each packet the network-layer address of each node along the explicit path. However, this approach generally makes the overhead in the packet prohibitively expensive. In MPLS, a path (known as a label switched path or LSP) is identified by a concatenation of labels which are stored in the nodes. As in traditional virtual-circuit packet switching, a packet is forwarded along the LSP by swapping labels. Thus, support of explicit routing in MPLS does not entail additional packet header overhead.

Several researchers have proposed to add traffic engineering capabilities in traditional datagram networks using shortest path algorithms (e.g., see [15], [9]). Although such schemes have been shown to improve the efficiency of the network, they suffer from several limitations including:

- load sharing cannot be accomplished among paths of different costs,

- traffic/policy constraints (for example, avoiding certain links for particular source-destination traffic) are not taken into account,

- modifications of link metrics to re-adjust traffic mapping tend to have network-wide effects causing undesirable and unanticipated traffic shifts, and

- traffic demands must be predictable and known a priori.

The combination of MPLS technology and its traffic engineering capabilities are expected to overcome these limitations. Explicit LSPs and flexible traffic assignment address the first limitation. Constraint-based routing has been proposed to address the second limitation. Furthermore, network-wide effects can be prevented when LSPs are pinned down. A change in LSP route will only cause the disturbance of the traffic for the corresponding source-destination pair. The objective of this paper is to address the last limitation.

In MPLS, traffic engineering mechanisms may be timedependent or state-dependent. In a time-dependent mechanism, historical information based on seasonal variations in traffic is used to pre-program LSP layout and traffic assignment. Additionally, customer subscription or traffic projection may be used. Pre-programmed LSP layout typically changes on a relatively long time scale (e.g., diurnal). Time-dependent mechanisms do not attempt to adapt to unpredictable traffic variations or changing network conditions. An example of a time-dependent mechanism is a global centralized optimizer where the input to the system is a traffic matrix and multiclass QoS requirements as described in [13].

When there are appreciable variations in actual traffic that could not predicted using historical information, a timedependent mechanism may not be able to prevent significant imbalance in loading and congestion. In such a situation, a statedependent mechanism can be used to deal with adaptive traffic assignment to the established LSPs according to the current state of the network which may be based on utilization, packet delay, packet loss, etc. In this paper, we assume that LSP layout has been determined using a long-term traffic matrix. The focus is on load balancing short-term traffic fluctuations among multiple 


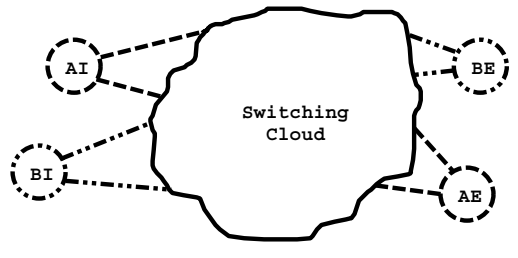

Fig. 1. A Transit Network Running MATE

LSPs between an ingress node and an egress node.

\section{B. Overview}

We propose a state-dependent traffic engineering mechanism called Multipath Adaptive Traffic Engineering (MATE). Some of the features of MATE include:

- distributed adaptive load-balancing algorithm,

- end-to-end control between ingress and egress nodes,

- no new hardware or protocol requirement in intermediate nodes,

- no knowledge of traffic demand is required,

- no assumption on the scheduling or buffer management schemes at a node,

- optimization decision based on path congestion measure,

- minimal packet reordering, and

- no clock synchronization between two nodes.

MATE operational setting assumes that that several explicit LSPs (typically range from two to five) between an ingress node and an egress node in an MPLS domain have been established using a standard protocol such as CR-LDP [10] or RSVP-TE [3], or configured manually. This is a typical setting which exists in an operational ISP network that implements MPLS. The goal of the ingress node is to distribute the traffic across the LSPs so that the loads are balanced and congestion is thus minimized. The traffic to be balanced by the ingress node is the aggregated flow (called traffic trunk in [12]) that shares the same destination. MATE is intended for traffic that does not require bandwidth reservation with best-effort traffic being the most dominant type. Figure 1 shows an example of a network environment where there are two ingress nodes, AI and BI, and two egress nodes, AE and BE, in an MPLS domain. MATE would be run on $\mathrm{AI}$ and $\mathrm{BI}$ to balance traffic destined to $\mathrm{AE}$ and $\mathrm{BE}$, respectively, across the LSPs connecting from AI to AE and from BI to BE. Note that the LSPs connecting the two pairs may be overlapping and sharing the same resources. However, this paper shows that stability can be guaranteed even though the pairs operate asynchronously.

Figure 2 shows a functional block diagram of MATE located at an ingress node. Incoming traffic enters into a filtering and distribution function whose objective is to facilitate traffic shifting among the LSPs in a way that reduces the possibilities of having packets arrive at the destination out of order. The mechanism does not need to know the statistics of the traffic demands or flow state information.

The traffic engineering function decides on when and how to shift traffic among the LSPs. This is done based on LSP statistics which are obtained from measurement using probe packets. The traffic engineering function consists of two phases: a monitoring phase and a load balancing phase. In the monitoring

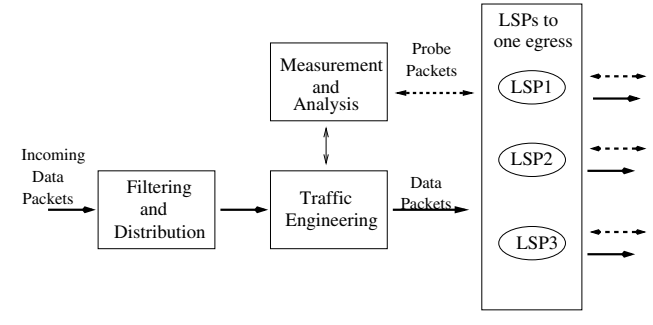

Fig. 2. MATE Functions in an Ingress Node

phase, if an appreciable and persistent change in the network state is detected, transition is made to the load balancing phase. In the load balancing phase, the algorithm tries to equalize the congestion measures among the LSPs. Once the measures are equalized, the algorithm moves to the monitoring phase and the whole process repeats.

The role of the measurement and analysis function is to obtain one-way LSP statistics such as packet delay and packet loss. This is done by having the ingress node transmit probe packets periodically to the egress node which returns them back to the ingress node. Probing may be done per class, i.e., probe packets have the same type of service header information as the traffic class being engineered. Based on the information in the returning probe packets, the ingress node is able to compute the oneway LSP statistics. Estimators of LSP statistics from the probes may be obtained reliably and efficiently using bootstrap resampling techniques. These techniques provide a dynamic mechanism for sending the probe packets so that the smallest number is automatically selected as the traffic conditions change to provide a given desirable degree of accuracy. Recent measurements in the Internet indicate little variations of aggregate traffic on links in 5-min intervals [17]. This quasi-stationarity condition where traffic statistics change relatively slowly (much longer than the round-trip delay between the ingress and egress nodes) facilitates traffic engineering and load balancing based on measurement of LSP statistics.

\section{Paper organization}

The rest of the paper is organized as follows. Section II presents an analytical model for multipath load balancing, derives a class of MATE algorithms, and proves their stability and optimality. Section III details the overall MATE scheme and discusses several implementation techniques, such as traffic filtering and distribution, traffic measurement, bootstrapping, etc. Section IV describes an experimental setup to verify the effectiveness of the proposed scheme. Section V presents the performance results and illuminates the behaviors of the algorithm in different networking environments and with traffic models ranging from Poisson to traffic models with longer dependence. The Appendix provides analytical support and proofs for the results presented.

\section{MATE ALGORITHMS}

In this section we present an analytical model of multipath load balancing, derive a class of asynchronous MATE algorithms, and prove their stability and optimality. 


\section{A. Model}

We model a MATE network by a set $L$ of unidirectional links. It is shared by a set $S$ of ingress-egress (IE) node pairs, indexed $1,2, \ldots, S$. Each of these IE pairs $s$ has a set $P_{s} \subseteq 2^{L}$ of LSPs available to it. Note that, by definition, no two (distinct) IE pair uses the same LSP, even though some of their LSPs may share links. Hence $P_{s}$ are disjoint sets.

An IE pair $s$ has a total input traffic of rate $r_{s}$ and routes $x_{s p}$ amount of it on LSP $p \in P_{s}$ such that

$$
\sum_{p \in P_{s}} x_{s p}=r_{s}, \quad \text { for all } s
$$

Let $x_{s}=\left(x_{s p}, p \in P_{s}\right)$ be the rate vector of $s$, and let $x=$ $\left(x_{s p}, p \in P_{s}, s \in S\right)$ the vector of all rates.

The flow on a link $l \in L$ has a rate that is the sum of source rates on all LSPs that traverse link $l$ :

$$
x^{l}=\sum_{s \in S} \sum_{l \in p, p \in P_{s}} x_{s p}
$$

Associated with each link $l$ is a cost $C_{l}\left(x^{l}\right)$ as a function of the link flow $x^{l}$. We assume that, for all $l, C_{l}(\cdot)$ is convex (and hence continuous).

Our objective is to minimize the total cost $C(x)=\sum_{l} C_{l}\left(x^{l}\right)$ by optimally routing the traffics on LSPs in $\cup_{s} P_{s}$ :

$$
\begin{array}{cl}
\min _{x} & C(x)=\sum_{l} C_{l}\left(x^{l}\right) \\
\text { subject to } & \sum_{p \in P_{s}} x_{s p}=r_{s}, \text { for all } s \in S \\
& x_{s p} \geq 0, \text { for all } p \in P_{s}, s \in S .
\end{array}
$$

A vector $x$ is called a feasible rate if it satisfies (2-3). A feasible rate $x$ is called optimal if it is a minimizer to the problem (1-3).

As observed in [5, Chapter 5], the derivative of the objective function with respect to $x_{s p}$ is

$$
\frac{\partial C}{\partial x_{s p}}(x)=\sum_{l \in p} C_{l}^{\prime}\left(x^{l}\right)
$$

We will interpret $C_{l}^{\prime}\left(x^{l}\right)$ as the first derivative length of link $l$, and $\partial C / \partial x_{s p}(x)$ as the (first derivative) length of LSP $p$.

The following characterization of optimal rate is a direct consequence of the Kuhn-Tucker theorem (see also [5, Chapter 5]). It says that at optimality a pair splits its traffic only among LSPs that have the minimum (and hence equal) first derivative lengths.

Theorem 1: The rate vector $x^{*}$ is optimal if and only if, for each pair $s$, all LSPs $p \in P_{s}$ with positive flows have minimum (and equal) first derivative lengths.

\section{B. Asynchronous algorithm}

A standard technique to solve the constrained optimization problem (1-3) is the gradient projection algorithm. In such an algorithm routing is iteratively adjusted in opposite direction of the gradient and projected onto the feasible space defined by (23). Each iteration of the algorithm takes the form:

$$
x(t+1)=[x(t)-\gamma \nabla C(t)]^{+}
$$

where $\gamma>0$ is a stepsize and should be chosen sufficiently small, $\nabla C(t)$ is a vector whose $(s, p)$ th element is the first derivative length $[\nabla C(t)]_{s p}=\partial C / \partial x_{s p}$ of LSP $p$ at time $t$, and $[z]^{+}$is the projection of a vector $z$ onto the feasible space. The algorithm terminates when there is no appreciable change, i.e., $\|x(t+1)-x(t)\|<\epsilon$ for some predefined $\epsilon$.

Note that the above iteration can be distributively carried out by each pair $s$ without the need to coordinate with other pairs:

$$
x_{s}(t+1)=\left[x_{s}(t)-\gamma \nabla C_{s}(t)\right]^{+}
$$

where $x_{s}(t)=\left(x_{s p}(t), p \in P_{s}\right)$ is $s$ 's rate vector at time $t$, and $\nabla C_{s}(t)=\left(\partial C / \partial x_{s p}(x(t)), p \in P_{s}\right)$ is the vector of first derivative lengths of LSPs in $P_{s}$. However (4) is not realistic, for two reasons.

First (4) assumes all updates are synchronized. Second it assumes zero feedback delay. Specifically (4) assumes that as soon as the IE pairs have calculated a new rate vector $x(t)$, it is reflected immediately in all the link flows:

$$
x^{l}(t)=\sum_{s} \sum_{l \in p, p \in P_{s}} x_{s p}(t)
$$

and in all the first derivative lengths:

$$
\frac{\partial C}{\partial x_{s p}}(x(t))=\sum_{l \in p} C_{l}^{\prime}\left(x^{l}(t)\right)
$$

Moreover all pairs $s$ have available these new values in $\nabla C_{s}(t)$ for computation of the rate vector in the next period. In practice the IE pairs update their rates asynchronously and in an uncoordinated manner. Moreover the first derivative length of a LSP can only be estimated empirically by averaging several measurements over a period of time. We now extend the model to take these factors into account.

Let $T_{s} \subseteq\{1,2, \ldots$,$\} be a set of times at which IE pair s$ adjusts its rate based on its current knowledge of the (first derivative) lengths of LSPs $p \in P_{s}$. At a time $t \in T_{s}, s$ calculates a new rate vector

$$
x_{s}(t+1)=\left[x_{s}(t)-\gamma \lambda_{s}(t)\right]^{+}
$$

and, starting from time $t+1$, splits its traffic $r_{s}$ along its LSPs in $P_{s}$ according to $x_{s}(t+1)$ until after the next update time in $T_{s}$. Here $\lambda_{s}(t)$ is an estimate of the first derivative length vector at time $t$, and is calculated as follows.

The new rates calculated by the IE pairs may be reflected in the link flows after certain delays. We model this by (cf. (5))

$$
\begin{array}{r}
\hat{x}^{l}(t)=\sum_{t^{\prime}=t-t_{0}}^{t} \sum_{s} \sum_{l \in p, p \in P_{s}} a_{l s p}\left(t^{\prime}, t\right) x_{s p}\left(t^{\prime}\right) \\
\sum_{t^{\prime}=t-t_{0}}^{t} a_{l s p}\left(t^{\prime}, t\right)=1, \quad \forall t, \forall l, s, p \in P_{s}
\end{array}
$$

In the above $\hat{x}^{l}(t)$ represents the flow rate available at link $l$ at time $t$ and is an weighted average (convex sum) of past source rates $x_{s p}\left(t^{\prime}\right)$. The weights $a_{l s p}\left(t^{\prime}, t\right)$ depend on $(l, s, p, t)$ and can be different between each source $s$ and link $l$, on different 
LSPs $p$, and at different times $t$. This model is very general and includes in particular the following two popular types:

- Latest data only: only the latest rate $x_{s p}(\tau)$, for some (typically unknown) $\tau \in\left\{t-t_{0}, \ldots, t\right\}$, is used in the measurement of $\hat{x}^{l}(t)$, i.e., $a_{l s p}\left(t^{\prime}, t\right)=1$ if $t^{\prime}=\tau$ and 0 otherwise.

- Latest average: only the average over the latest $k$ rates is used in the measurement of $\hat{x}^{l}(t)$, i.e., $a_{l s p}\left(t^{\prime}, t\right)>0$ for $t^{\prime}=\tau-$ $k+1, \ldots, \tau$ and 0 otherwise, for some (typically unknown) $\tau \in$ $\left\{t-t_{0}, \ldots, t\right\}$.

An IE pair $s$ estimates the first derivative length of an LSP $p \in P_{s}$ by asynchronously collecting a certain number of measurements (using probe packets, see below), and forming their mean. Hence (cf. (6))

$$
\begin{gathered}
\lambda_{s p}(t)=\sum_{t^{\prime}=t-t_{0}}^{t} \sum_{l \in p} b_{l s p}\left(t^{\prime}, t\right) C_{l}^{\prime}\left(\hat{x}^{l}\left(t^{\prime}\right)\right) \\
\sum_{t^{\prime}=t-t_{0}}^{t} b_{l s p}\left(t^{\prime}, t\right)=1, \quad \forall t, \forall l, s, p \in P_{s}
\end{gathered}
$$

Again the estimate is obtained by 'averaging' over the past values of LSP lengths, and can depend on $(l, s, p, t)$. The model is very general and include the special cases of using only the last received measurement or the average over the last $k$ values, as discussed above. The interpretation in both cases is that the measurements $\sum_{l \in p} C_{l}^{\prime}\left(\hat{x}^{l}\left(t^{\prime}\right)\right)$ for $t^{\prime}>\tau$ have not been received by $s$ by time $t$, and the measurements for $t^{\prime}<\tau$ (latest data only) or for $t^{\prime} \leq \tau-k$ (latest average) have been discarded.

This concludes the description of our algorithm model (equations 7-11). The model is similar to that in [18], with two differences. First their model distinguishes between the desired rate $x(t)$ as calculated by the projection algorithm and the actual realized source rate $\hat{x}(t)$. The actual rate $\hat{x}(t)$ is a convex combination of the current desired rate $x(t)$ and the previous actual rate $\hat{x}(t-1)$. This models the fact that a desired rate $x(t)$ may not be realized immediately, as in a virtual circuit network where virtual circuits may persist over several update cycles. We are however only dealing with IP datagrams and hence it is reasonable to assume that each ingress node can shift its traffic among the LSPs available to it immediately after each update. Second their model assumes that, at time $t$, each $s$ has available the current first derivative lengths $\sum_{l \in p} C^{\prime}\left(\hat{x}^{l}(t)\right)$ and uses it in place of the gradient in the update algorithm. We however assume that, at time $t, s$ may only have outdated first derivative lengths (see (10-11)); moreover $s$ uses a weighted average over several past lengths in the update algorithm. This is because, in our case, $s$ can only estimate the first derivative lengths through noisy measurement.

The next result states that the algorithm converges to an optimal routing, provided the following conditions are satisfied:

C1: The cost functions $C_{l}(z)$ are twice continuously differentiable and convex.

C2: Their derivatives $C_{l}^{\prime}(z)$ are Lipschitz over any bounded sets, i.e., for any bounded set $B_{l} \subset \Re$ there exists a constant $c_{l}$ such that for all $z, z^{\prime} \in B_{l}$, we have $\left|C_{l}^{\prime}(z)-C_{l}^{\prime}\left(z^{\prime}\right)\right| \leq c_{l}\left|z-z^{\prime}\right|$. C3: For any constant $c$ the sets $\left\{z \mid C_{l}(z) \leq c\right\}$ are bounded.

C4: The time interval between updates is bounded.

Theorem 2: Under conditions $\mathrm{C} 1-\mathrm{C} 4$, starting from any initial vector $x(0)$, there exists a sufficiently small stepsize $\gamma$ such that any accumulation point of the sequence $\{x(t)\}$ generated by the asynchronous algorithm is optimal.

A more careful accounting shows that the stepsize $\gamma$, and hence the speed of convergence, depends on the degree of asynchronism as expressed by the parameter $t_{0}$ defined in (8), the 'steepness' of the cost function as expressed by the Lipschitz constant in condition $\mathrm{C} 2$, and the size of the network. For ease of exposition, suppose the cost functions are uniformly globally Lipschitz, i.e., for all links $l$ and all $z, z^{\prime}$, we have

$$
\left|C_{l}^{\prime}(z)-C_{l}^{\prime}\left(z^{\prime}\right)\right| \leq L\left|z-z^{\prime}\right|
$$

Theorem 3: An upper bound in Theorem 2 is:

$$
\gamma<\frac{1}{L\left(1+\pi h \lambda\left(2 t_{0}+1\right)\right)}
$$

where $\pi$ is the total number of LSPs in the network, $h$ is the number of hops in the longest (maximum-hop) LSP, $\lambda$ is the maximum number of LSPs going through a link, and $t_{0}$, defined in (8), measures the degree of asynchronism.

The theorem suggests that the larger the degree of asynchronism measured by $t_{0}$, the smaller the stepsize and hence slower the convergence.

\section{Example cost function}

The choice of cost functions determines the parameters to be measured and equalized in carrying out MATE. A natural choice for the link cost is delay. Then Theorem 1 implies that the derivatives of the $L S P$ delay are the congestion measures to be equalized.

If we take the delay to be the average delay of an $M / M / 1$ queue $C_{l}\left(x^{l}\right)=1 /\left(\mu_{l}-x^{l}\right), \mu_{l}$ representing the link capacity then $C_{l}$ satisfies the conditions of Theorem 2 and hence the algorithm will converge to an optimal traffic split. Note that the link capacity $\mu_{l}$ typically fluctuates randomly. Hence the delay derivatives cannot be computed and must be measured. Loss may be incorporated into the cost by treating each packet loss as a fixed (large) delay. Another alternative is to use the product of loss and delay as the cost function. In summary, the basic goal is to steer the network towards a desired operating performance based on a chosen cost function.

\section{MATE IMPLEMENTATION TECHNIQUES}

In this section, we provide further elaboration on the techniques employed in our implementation of the MATE functions.

\section{A. Traffic filtering and distribution}

The traffic filtering and distribution function first distributes the traffic to be engineered for a given ingress-egress pair equally among $N$ bins, where the number of bins determines the minimum amount of the traffic that can be shifted. If the total incoming traffic to be engineered is of rate $R \mathrm{bps}$, each bin would receive an amount of $r=R / N$ bps. The traffic from the $N$ bins is then mapped into the $M$ LSPs according to the MATE algorithm described in the last section.

The engineered traffic can be filtered and distributed into the $N$ bins in a number of ways. A simple method is to distribute the traffic on a per-packet basis without filtering. For example, one 
may distribute incoming packets at the ingress node to the bins in a round-robin fashion. Although it does not have to maintain any per-flow state, the method suffers from potentially having to reorder an excessive amount of packets for a given flow which is undesirable for TCP applications.

On the other extreme, one may filter the traffic on a per-flow basis (e.g., based on < source IP address, source port, destination IP address, destination port, IP protocol $>$ tuple), and distribute the flows to the bins such that the loads are similar. Although per-flow traffic filtering and distribution preserves packet sequencing, this approach has to maintain a large number of states to keep track of each active flow.

Another method is to filter the incoming packets by using a hash function on the IP field(s). The fields can be based on the source and destination address pair, or other combinations. A typical hash function is based on a Cyclic Redundancy Check (CRC). The purpose of the hash function is to randomize the address space to prevent hot spots. Traffic can be distributed into the $N$ bins by applying a modulo-N operation on the hash space. Note that packet sequence for each flow is maintained with this method.

After the engineered traffic is distributed into the $N$ bins, a second function maps each bin to the corresponding LSP according to the MATE algorithm. The rule for the second function is very simple. If LSP $i$ is to receive twice as much traffic as LSP $j$, then LSP $i$ should receive traffic from twice as many bins as LSP $j$. The value $N$ should be chosen so that the smallest amount of traffic that can be shifted, which is equal to $1 / N$ of the total incoming traffic, has a reasonable granularity.

\section{B. Traffic measurement and analysis}

The efficacy of any state-dependent traffic engineering scheme depends crucially on the traffic measurement process. MATE does not require each node to perform traffic measurement. Only the ingress and egress nodes are required to participate in the measurement process.

For the purpose of balancing the loads among LSPs, the available bandwidth appears to be a desirable metric to measure. The methods for measuring the available bandwidth of a given path have been described in the past (e.g., see [11], [8]). Based on our experience, this metric turns out to be difficult to measure accurately using the minimal requirements assumed in MATE.

To this end, we found that packet delay is a metric that can be reliably measured. The delay of a packet on an LSP can be obtained by transmitting a probe packet from the ingress node to the egress node. The probe packet is time-stamped at the ingress node at time $T_{1}$ and recorded at the egress node at time $T_{2}$. If the ingress' clock is faster than the egress' clock by $T_{d}$, then the total packet delay (i.e, queueing time, propagation time, and processing time) is $T_{2}-T_{1}+T_{d}$. A group of probe packets sent one at a time on an LSP can easily yield an estimate of the mean packet delay $E\left[T_{2}-T_{1}\right]+T_{d}$. The reliability of the estimator can be evaluated by bootstrapping (see details below) to give the confidence interval for the mean delay. One important point to note is that the value of $T_{d}$ is not required when only the marginal delay is needed. MATE exploits this property by relying only on marginal delays rather than absolute delays. Therefore, clock synchronization is not necessary.
Packet loss probability is another metric that can be estimated by a group of probe packets. In general, only reasonably high packet loss rates can be reliably observed. Packet loss probability can be estimated by encoding a sequence number in the probe packet to notify the egress node how many probe packets have been transmitted by the ingress node, and another field in the probe packet to indicate how many probe packets have been received by the egress node. When a probe packet returns, the ingress node is able to estimate the one-way packet loss probability based on the number of probe packets that has been transmitted and the number that has been received. The advantage of this approach is that it is resilient to losses in the reverse direction.

The bootstrap is a powerful technique for assessing the accuracy of a parameter estimator in situations where conventional techniques are not valid [19]. Most other techniques for computing the variance of parameter estimators or for setting confidence intervals for the true parameter assume that the size of the available set of sample values is sufficiently large, so that asymptotic results (central limit theorem) can be applied. However, in many situations the sample size is necessarily limited, such is the case in traffic engineering mechanisms like MATE, where the probe packet load should not consume significant network resources. In MATE, we can use the bootstrap to obtain reliable estimates of the congestion measures of the mean delay and cell loss rate from a given set of measurements obtained via the probe packets. By selecting a desirable confidence interval, we get a dynamic way of specifying the number of observations needed. This provides a built-in reliability estimator which automatically selects the required number of probe packets to send. We have found this quite useful in our implementations, in comparison with schemes where the number of probe packets is set in an ad-hoc manner, and the number of probes may be too small or too large. The following is a basic procedure for computing a confidence interval:

- Step 0: Suppose the original sample is $X=\left\{x_{1}, x_{2}, \ldots, x_{m}\right\}$.

- Step 1: Draw a random sample of $m$ values, with replacement, from $X$. This produces the bootstrap resample $Y$.

- Step 2: Calculate the mean for $Y$ (say, $\mu_{1}$ ).

- Step 3: Repeat steps 1 and 2 a large number of times to obtain n bootstrap estimates $\mu_{1}, \mu_{2}, \ldots, \mu_{n}$.

- Step 4: Sort the bootstrap estimates into increasing order $\mu(1), \ldots, \mu(n)$.

- Step 5: The desired $(1-\alpha) 100 \%$ bootstrap confidence interval for the mean is $\left(\mu\left(q_{1}\right), \mu\left(q_{2}\right)\right)$, where $q_{1}=(n \alpha / 2)$ and $q_{2}=$ $n-q_{1}+1$.

\section{EXPERIMENTAL METHODOLOGY}

In this section, we use simulations to evaluate the effectiveness of MATE. The objective of our simulation study is to show that within a network that has multiple LSPs between some ingress and egress nodes, the traffic distribution under the MATE algorithm is stable, and load balancing is achieved. We concentrate on two network topologies: one with a single ingress-egress pair connected by multiple LSPs, and the other with multiple ingress-egress pairs where some links are shared among the LSPs from different pairs. Note that in the latter case, there is a considerable interaction between the pairs. In the fol- 


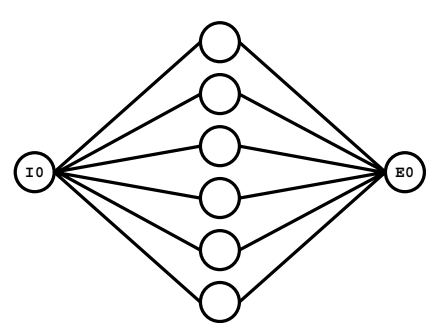

Fig. 3. Experiment Network Topology 1

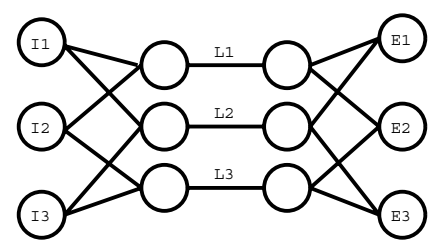

Fig. 4. Experiment Network Topology 2

lowing, we will present a description of the simulator, the test networks we use, and the data collection.

We wrote a packet level discrete-event simulator, which supports entities such as packet queues, switched LSPs, network connections. We consider networking environments where the traffic conditions vary due to changes in network load (link utilization), for example, due to "rush hour" conditions, or some LSP failures, and traffic variations due to correlations and dependencies. We realize that we can not distribute packets belonging to short lived network connections. As a result, we specify two types of traffic in our simulator: engineered traffic and cross traffic. The engineered traffic is the traffic that needs to be balanced, and the cross traffic is the background traffic that we have no control over. We assign a lifetime to each traffic source so we are able to simulate the dynamic behaviors of a network by switching on and off cross traffic sources. We consider a traffic model which exhibits short-range dependencies, such as Poisson, and another model which can be tuned to model a large degree of dependencies. For the latter we use the $\operatorname{DAR}(p)$ process (discrete autoregressive process of order $p$ ) [16]. The parameter $p$ determines the time-scale over which traffic dependency and correlation are exhibited. If $p$ is 1 , the process is a standard Markov process. In our experiments we set $p$ to a value of 10; this leads to a substantial degree of correlation in the generated traces.

Figure 3 and Figure 4 are the two network topologies used in our experiment. The first topology consists of a single pair of ingress-egress nodes. There are 6 LSPs connecting the ingress node to the egress node, and all links are identical so that the LSPs have the same bottleneck link bandwidth.

In the second network topology, we have 3 ingress nodes, I1, I2, and I3, and three egress nodes, E1, E2, and E3. Altogether, they form three pairs. The links in this network are again all identical. Each ingress-egress pair has two LSPs for traffic balancing. We set up this scenario so there is a common link for every two pairs. In each of our simulations, the engineered traffic for each pair flows from the ingress node to the egress node. The cross traffic enters at the intermediate node and exits at egress node(s). We consider two implementations of the

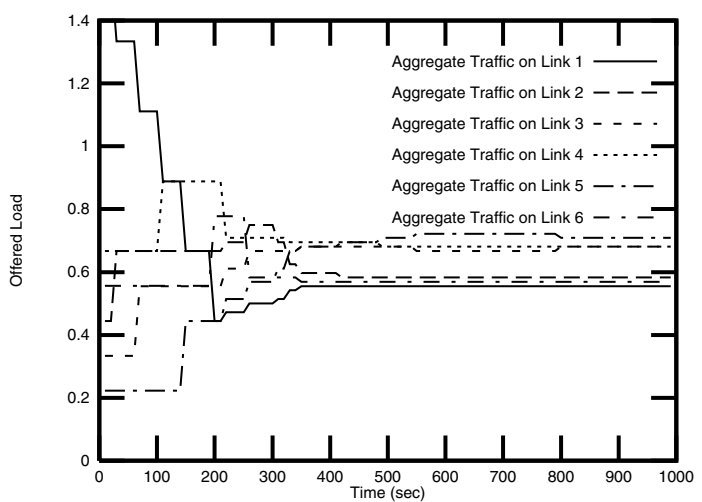

Fig. 5. Offered load under Poisson traffic for network topology 1

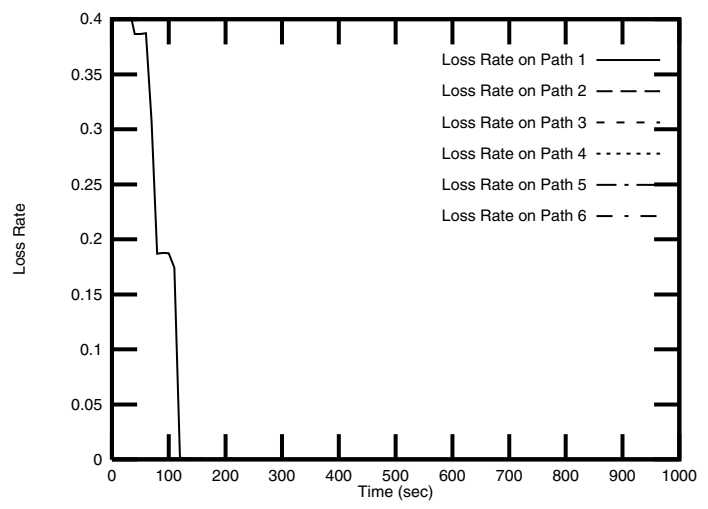

Fig. 6. Loss under Poisson traffic for network topology 1

basic algorithm. In the first one, a small random delay is introduced before the algorithm moves from the monitoring phase to the traffic engineering phase upon detection of change in traffic conditions. This damping mechanism reduces synchronization among multiple ingress nodes. In the second implementation, there is a coordination among the ingress nodes so that only one ingress node at a time enters the traffic engineering phase. This obviously requires a special coordination protocol. We omit the details in this paper.

In order to do data collection, we record the total offered load and the loss rate on each link. We compute the loss rate on each LSP from the link loss rates. The loss rate on an LSP can be computed by assuming that the link loss rates are independent as follows:

$$
\text { total_loss }=\left(1-\prod\left(1-\text { los }_{i}\right)\right)
$$

where the product is taken over all links $i$ in the LSP.

\section{Simulation RESUlts}

In this section, we show the results from the simulation of the two networks in the previous section. These results are encouraging in that they show our algorithms have good stability and convergence properties.

First we present the results from a single ingress-egress pair. We show two sets of data for this scenario. Figure 5 and Figure 6 show the results of an experiment with Poisson traffic on the network in Figure 3. Initially, all of the engineered traffic streams are routed on one of the LSPs, and cross traffic enter the 


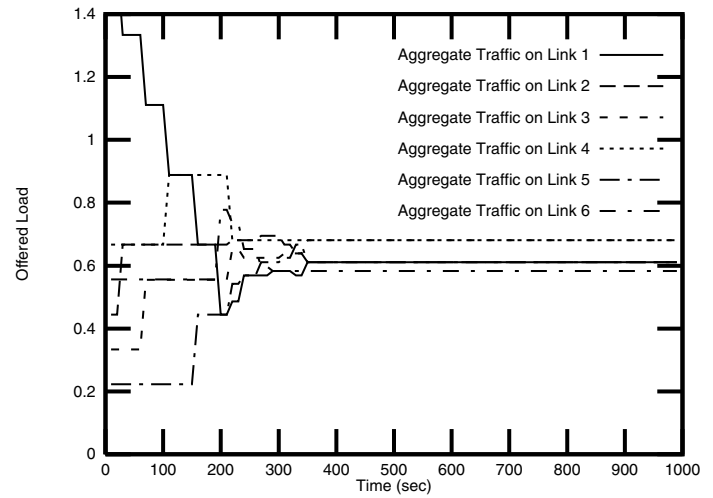

Fig. 7. Offered load under DAR traffic for network topology 1

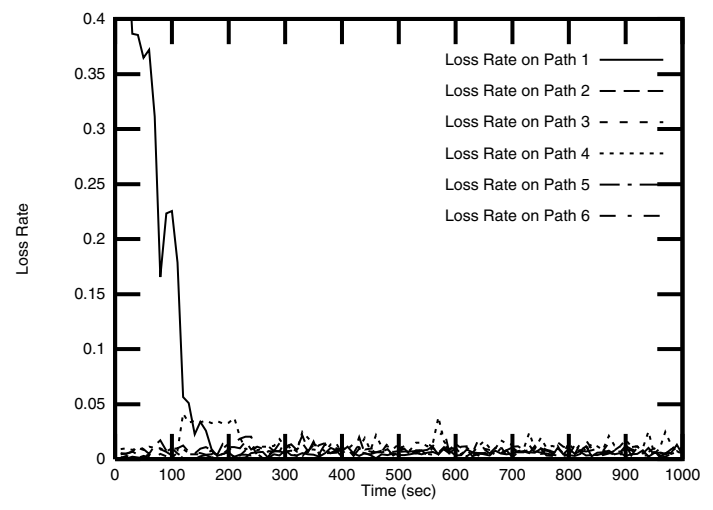

Fig. 8. Loss under DAR traffic for network topology 1

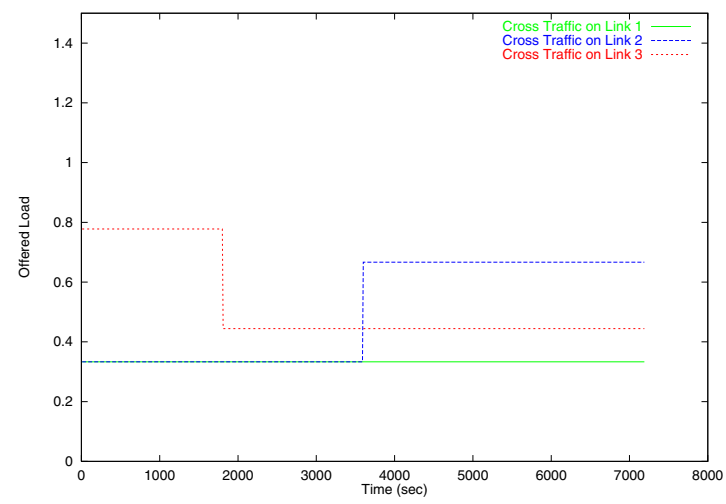

Fig. 9. Cross Traffic for network topology 2

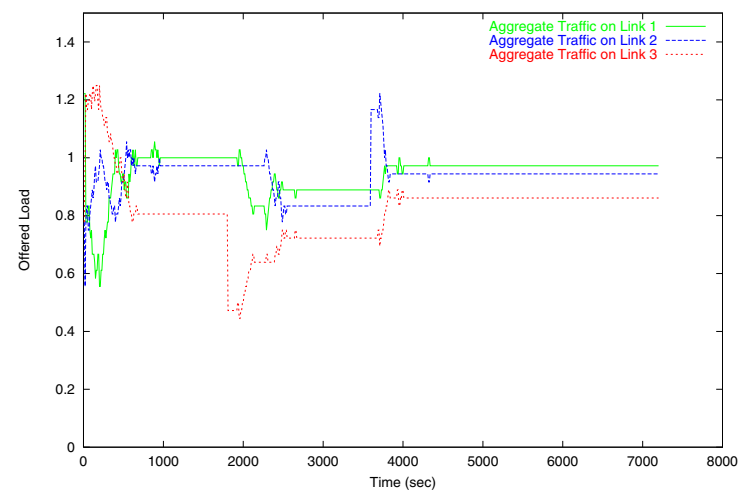

Fig. 10. Offered load under Poisson traffic for network topology 2

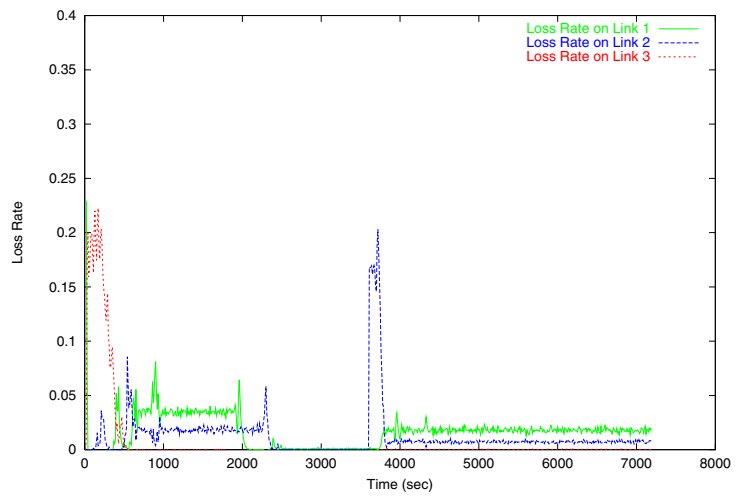

Fig. 11. Loss under Poisson traffic for network topology 2

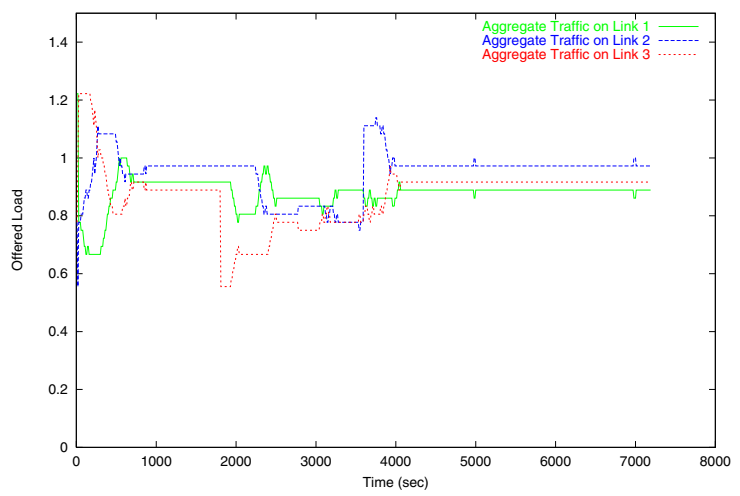

Fig. 12. Offered load under Poisson traffic with coordination for network topology 2

network at the intermediate nodes connecting the ingress and egress nodes. We have an unbalanced situation with one heavily congested LSP and five lightly loaded LSPs. As shown in the plot, the algorithm is able to successfully reduce the engineered traffic from the overloaded link and distribute them to the underutilized links. The loss curve shows clearly that the loss rate on the first LSP dropped from $40 \%$ to a value that is too small to observe $^{1}$. The loss rates on the other LSPs are maintained at negligible levels throughout the simulation. The final traffic distribution converges to a steady state, where utilizations are very

\footnotetext{
${ }^{1}$ Note that loss rates on the order of $10 \%$ to $20 \%$ are not atypical in the Inter-
} net.

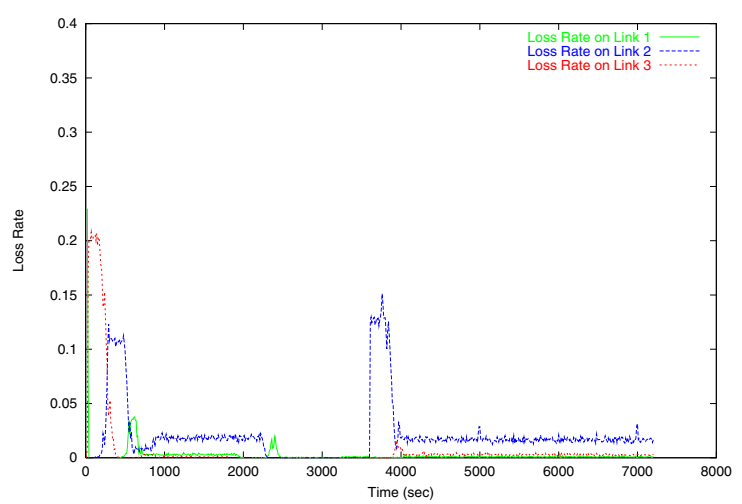

Fig. 13. Loss under Poisson traffic with coordination for network topology 2 


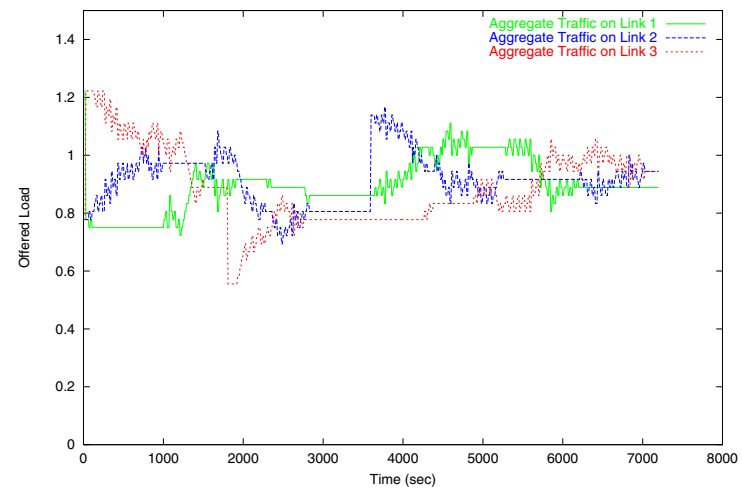

Fig. 14. Offered load under DAR traffic with coordination for network topology 2

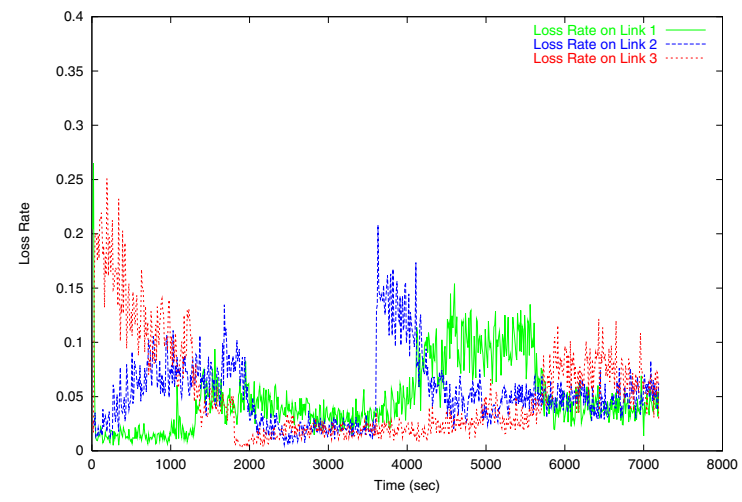

Fig. 15. Loss under DAR traffic with coordination for network topology 2

close on all LSPs. We observe similar behavior in Figures 7 and 8 where the Poisson streams are here replaced with DAR traffic streams that possess correlation and dependence. We point out that the probe traffic required in the each phase of the algorithm is around $0.5 \%$ of the engineered traffic, thereby ensuring the scalability of the overall approach.

The Figures 10 - 13 show the simulation scenario for Figure 4 under the two implementations mentioned earlier. Again the engineered traffic streams travel from the ingress node to the egress node, and the cross traffic enters through the intermediate nodes and exit at the egress nodes. The cross traffic dynamics are shown in Figure 9. There is a decrease in cross traffic on link 3 right before 2000 seconds and a increase in cross traffic on link 2 around 3600 seconds. In order to balance traffic, the algorithms must shift traffic into link 3 and possibly out of link 2. Both implementations essentially achieve the same performance, where utilizations and loss rates on three LSPs are comparable. Figure 14 and Figure 15 show the same simulation with DAR traffic instead of Poisson traffic where coordination among ingress node is considered.

\section{CONCLUSIONS}

Our focus on this paper was to apply adaptive traffic engineering to utilize network resource more efficiently and minimize congestion. We have proposed a class of algorithms called MATE, which tries to achieve these objectives using minimal assumptions through a combination of techniques such as bootstrap probe packets, which control the amount of extra traffic, and marginal delays that are easily measurable and do not require clock synchronization. Our analytical models prove the stability and optimality of MATE. Our simulation results show that MATE can effectively remove traffic imbalances among that may occur among multiple LSPs. We observe that, in many cases, high packet loss rates can be significantly reduced by properly shifting some traffic to less loaded LSPs. This should benefit many applications such as TCP. For future work we will consider more realistic networking environments and examine the impact of MATE on the application level.

\section{REFERENCES}

[1] D. O. Awduche, J. Malcolm, J. Agogbua, M. O’Dell, and J. McManus, "Requirements for traffic engineering over mpls", RFC 2702, Sep. 1999.

[2] D. Awduche, A. Chui, A. Elwalidn, I. Widjaja, and X. Xiao, "A framework for Internet traffic engineering", Internet draft $<$ draft-ietf-tewgframework-01.txt>, Mar. 2000

[3] D. O. Awduche et. al., "RSVP-TE: extensions to RSVP for LSP tunnels", Internet draft $<$ draft-ietf-mpls-rsvp-lsp-tunnel-05.txt $>$, Feb. 2000.

[4] D. Bertsekas, Nonlinear programming, Athena Scientific, 1995.

[5] D. Bertsekas and R. Gallager, Data networks, Prentice-Hall Inc., 2nd ed. edition, 1992.

[6] Dimitri P. Bertsekas and John N. Tsitsiklis, Parallel and distributed computation, Prentice-Hall, 1989.

[7] R. Callon, P. Doolan, N. Feldman, A. Fredette, G. Swallow, and A. Viswanathan, "A framework for multiprotocol label switching", Internet draft < draft-ietf-mpls-framework-05.txt>, Sep. 1999.

[8] R.L. Carter and M.E. Crovella, "Measuring bottleneck link speed in packet-switched networks", Technical Report BU-CS-96-006, Boston University, Mar. 1996.

[9] B. Fortz and M. Thorup, "Internet traffic engineering by optimizing OSPF weights", Proceedings of INFOCOM'2000, Tel-Aviv, Israel, Mar. 2000.

[10] B. Jamousi et. al., "Constraint-based LSP setup using LDP", Internet draft $<$ draft-ietf-mpls-cr-ldp-03.txt>, Sep. 1999.

[11] S. Keshav, "A control theoretic approach to flow control", Proceedings of SIGCOMM'91, ACM, August 1991.

[12] T. Li and Y. Rekhter, "Provider architecture for differentiated services and traffic engineering (PASTE)", RFC 2430, Oct. 1998.

[13] D. Mitra and K.G. Ramakrishnan, "A Case Study of Multiservice, Multipriority Traffic Engineering Design for Data Networks", Proc. Globecom'99, Dec 1999.

[14] E. C. Rosen, A. Viswanathan, and R. Callon, "Multiprotocol label switching architecture", Internet draft < draft-ietf-mpls-arch-05.txt>, Sep. 1999.

[15] M. A Rodrigues and K. G. Ramakrishnan, "Optimal routing in shortestpath networks", ITS '94, Rio de Genero, Brazil.

[16] B. K. Ryu and A. Elwalid, "The importance of long-range dependence of VBR video traffic in ATM traffic engineering", In Proceedings of SIGCOMM'96, pages 3-14, Aug. 1996.

[17] K. Thompson, G.J. Miller, and R. Wilder, "Wide-area internet traffic patterns and characteristics", IEEE Networks, 6(6), Dec. 1997.

[18] John N. Tsitsiklis and Dimitri P. Bertsekas, "Distributed asynchronous optimal routing in data networks", IEEE Transactions on Automatic Control, 31(4):325-332, April 1986.

[19] A. M. Zoubir and B. Boashash, "The bootstrap and its applications in signal processing", IEEE Signal Processing Magazine, Jan. 1998.

\section{APPENDIX}

\section{Proof of Theorem 1}

Since the cost function is convex the first order optimality condition is both necessary and sufficient: $x^{*}$ is optimal if and only if $x^{*}$ is feasible and there exist constants $\xi_{s}$ such that for all $(s, p)$

$$
\frac{\partial C}{\partial x_{s p}}\left(x^{*}\right)=\sum_{l \in p} C_{l}^{\prime}\left(x^{* l}\right) \geq \xi_{s}
$$

with equality if $x_{s p}^{*}>0$. Hence all LSPs $p \in P_{s}$ with $x_{s p}^{*}>0$ have their first derivative lengths equal to $\xi_{s}$. 


\section{Proof of Theorem 2}

Its proof is adapted from that in [18]. Let $z(t)=x(t+1)-$ $x(t)$. Using a first order Taylor expansion for $C$ we have for some rate vector $y(t)^{2}$

$$
\begin{aligned}
C(x(t+1))= & C(x(t))+\nabla C(x(t)) z(t)+ \\
& \frac{1}{2} z(t) \nabla^{2} C(y(t)) z(t) \\
\leq & C(x(t))+\lambda(t) z(t) \\
& +\|\nabla C(x(t))-\lambda(t)\| \cdot\|z(t)\| \\
& +A_{1}\|z(t)\|^{2}
\end{aligned}
$$

where $\lambda(t)=\left(\lambda_{s}(t), s \in S\right)$ and the constant $A_{1}$ depends on the initial vector $x(0)$. We next show that

$$
\begin{array}{r}
\lambda(t) z(t) \leq-\frac{1}{\gamma}\|z(t)\|^{2} \\
\|\nabla C(x(t))-\lambda(t)\| \cdot\|z(t)\| \leq A_{2} \sum_{t^{\prime}=t-2 t_{0}}^{t}\left\|z\left(t^{\prime}\right)\right\|^{2}
\end{array}
$$

for some constant $A_{2}$ that depends on $x(0)$.

First, note that (14) holds if the following holds for all $s$ :

$$
\lambda_{s}(t) z_{s}(t) \leq-\frac{1}{\gamma}\left\|z_{s}(t)\right\|^{2}
$$

For $t \notin T_{s}$ (16) trivially holds. For $t \in T_{s}$ apply the projection theorem [4] to (7) to obtain

$$
(x(t)-\gamma \lambda(t)-x(t+1))(x(t)-x(t+1)) \leq 0
$$

Rearranging terms yields (16).

To show (15) note that since all norms in $\Re^{n}$ are equivalent there exist constants $A_{3}$ and $A_{4}$ such that

$$
\begin{aligned}
& || \nabla C(t)-\lambda(t)||_{2} \\
\leq & A_{3} \max _{s} \max _{p \in P_{s}}\left|\frac{\partial C}{\partial x_{s p}}(x(t))-\lambda_{s p}(t)\right| \\
\leq & A_{3} \max _{s} \max _{p \in P_{s}} \sum_{l \in p} \mid C_{l}^{\prime}\left(x^{l}(t)\right) \\
& -\sum_{t^{\prime}=t-t_{0}}^{t} b_{l s p}\left(t^{\prime}, t\right) C_{l}^{\prime}\left(\hat{x}^{l}\left(t^{\prime}\right)\right) \mid \\
\leq \quad & A_{4} \max _{s} \max _{p \in P_{s}} \max _{l \in p} \max _{t-t_{0} \leq t^{\prime} \leq t} \\
& \left|C_{l}^{\prime}\left(x^{l}(t)\right)-C_{l}^{\prime}\left(\hat{x}^{l}\left(t^{\prime}\right)\right)\right| \\
= & A_{4} \max _{l \in L} \max _{t-t_{0} \leq t^{\prime} \leq t}\left|C_{l}^{\prime}\left(x^{l}(t)\right)-C_{l}^{\prime}\left(\hat{x}^{l}\left(t^{\prime}\right)\right)\right|
\end{aligned}
$$

Let $B:=\{x \mid C(x) \leq C(x(0))\}$ and $B_{l}:=\{f \mid f=$ $\sum_{s} \sum_{l \in p, p \in P_{s}} x_{s p}$, for some $\left.x \in B\right\}$. In words, $B$ is the set of rate vectors $x$ at which the total cost $C(x)$ is no greater than the initial cost. As will be seen, provided the stepsize $\gamma$ is sufficiently small, $C(x(t)) \leq C(x(0))$ for all $t$ (see (21)). That is, $B$ is the set of all possible rate vectors given the initial $x(0)$. (This

\footnotetext{
${ }^{2}$ For simplicity we write $x y$ instead of the more correct notation $x^{T} y$ for the inner product of two vectors $x$ and $y$. We usually use $\|x\|$ to denote the Euclidean norm, but sometimes $\|x\|_{2}$ for emphasis.
}

can be made more rigorous by induction.) Then $B_{l}$ is the set of all possible link flows on link $l$. By condition $\mathrm{C} 2$, we have for some constants $A_{5}, A_{6}, A_{2}$

$$
\begin{aligned}
& || \nabla C(t)-\lambda(t) \|_{2} \\
\leq & A_{5} \max _{l \in L} \max _{t-t_{0} \leq t^{\prime} \leq t}\left|x^{l}(t)-\hat{x}^{l}\left(t^{\prime}\right)\right| \\
\leq & A_{5} \max _{l \in L} \max _{t-t_{0} \leq t^{\prime} \leq t} \sum_{s} \sum_{l \in p, p \in P_{s}} \\
& \left|x_{s p}(t)-\sum_{t^{\prime \prime}=t^{\prime}-t_{0}}^{t^{\prime}} a_{l s p}\left(t^{\prime \prime}, t^{\prime}\right) x_{s p}\left(t^{\prime \prime}\right)\right| \\
\leq & A_{6} \max _{s} \max _{p \in P s} \max _{t-t_{0} \leq t^{\prime} \leq t} \max _{t^{\prime}-t_{0} \leq t^{\prime \prime} \leq t^{\prime}}\left|x_{s p}(t)-x_{s p}\left(t^{\prime \prime}\right)\right| \\
\leq & A_{6} \max _{s} \max _{p \in P_{s}}\left|x_{s p}(t)-x_{s p}(t-1)\right|+\cdots \\
& +\left|x_{s p}\left(t-2 t_{0}+1\right)-x_{s p}\left(t-2 t_{0}\right)\right| \\
\leq & A_{2} \sum_{t^{\prime}=t-2 t_{0}}^{t-1} \| z\left(t^{\prime}\right)||_{2}
\end{aligned}
$$

Hence

$$
\begin{aligned}
& \|\nabla C(x(t))-\lambda(t)\| \cdot\|z(t)\| \\
\leq & A_{2} \sum_{t^{\prime}=t-2 t_{0}}^{t-1}\left\|z\left(t^{\prime}\right)\right\| \cdot\|z(t)\| \\
\leq & A_{2} \sum_{t^{\prime}=t-2 t_{0}}^{t}\left\|z\left(t^{\prime}\right)\right\|^{2}
\end{aligned}
$$

where the last inequality follows from the fact that the convex function $\sum_{i} y_{i} y+y^{2}-\sum_{i} y_{i} y$ attains its minimum of zero over $\left\{y_{i}, y \mid y_{i} \geq 0, y \geq 0\right\}$ at the origin. This completes the proof of (15).

Substituting (14-15) into (13) we have

$$
\begin{aligned}
& C(x(t+1)) \\
\leq & C(x(t))-\left(\frac{1}{\gamma}-A_{1}\right)\|z(t)\|^{2} \\
& +A_{2} \sum_{t^{\prime}=t-2 t_{0}}^{t}\left\|z\left(t^{\prime}\right)\right\|^{2}
\end{aligned}
$$

Summing over all $t$ we have

$$
\begin{aligned}
& C(x(t+1)) \\
\leq & C(x(0))-\left(\frac{1}{\gamma}-A_{1}\right) \sum_{\tau=0}^{t}\|z(\tau)\|^{2} \\
& +A_{2} \sum_{\tau=0}^{t} \sum_{t^{\prime}=\tau-2 t_{0}}^{\tau}\left\|z\left(t^{\prime}\right)\right\|^{2} \\
\leq & C(x(0))-\left(\frac{1}{\gamma}-A_{1}-A_{2}\left(2 t_{0}+1\right)\right) \\
& \sum_{\tau=0}^{t}\|z(\tau)\|^{2}
\end{aligned}
$$

Choose $\gamma$ small enough such that $\frac{1}{\gamma}-A_{1}-A_{2}\left(2 t_{0}+1\right)>0$. Since $x(t)$ is in a compact set and $C$ is continuous, $C(x(t))$ is 
lower bounded. Then since $C(x(t))$ is bounded for all $t$ we must have $\sum_{\tau=0}^{\infty}\|z(\tau)\|^{2}<\infty$, which implies

$$
\|z(t)\| \rightarrow 0 \quad \text { as } t \rightarrow \infty
$$

Substituting this into (20) we conclude that

$$
\lambda(t) \rightarrow \nabla C(t) \quad \text { as } t \rightarrow \infty
$$

Let $x^{*}$ be an accumulation point of $\{x(t)\}$. One exists since $\{x(t)\}$ is in a compact set. By (23) and the fact that $C$ is continuously differentiable we have

$$
\lambda(t) \rightarrow \lim _{t \rightarrow \infty} \nabla C(t)=\nabla C\left(x^{*}\right)
$$

Since the time interval between updates is bounded, for any $s$, we can find a subsequence $\left\{x\left(t_{k}\right), t_{k} \in T_{s}\right\}$ that converges to $x^{*}$, i.e., $\lim _{k} x\left(t_{k}\right)=x^{*}$. Applying again the projection theorem [4] to (7) we have for any feasible $x_{s}$

$$
\begin{aligned}
\left(x_{s}\left(t_{k}\right)-\gamma \lambda_{s}\left(t_{k}\right)-x_{s}\left(t_{k}+1\right)\right)\left(x_{s}-x_{s}\left(t_{k}+1\right)\right) & \leq 0 \\
\left(z_{s}\left(t_{k}\right)+\gamma \lambda_{s}\left(t_{k}\right)\right)\left(x_{s}-x_{s}\left(t_{k}+1\right)\right) & \geq 0
\end{aligned}
$$

Taking $k \rightarrow \infty$ we have by (22) and (24) that for any feasible $x_{s}$,

$$
\nabla C_{s}\left(x_{s}^{*}\right)\left(x_{s}-\lim _{k \rightarrow \infty} x_{s}\left(t_{k}+1\right)\right) \geq 0
$$

Since $z(t)=x(t+1)-x(t) \rightarrow 0$ by (22), we have $\lim _{k} x_{s}\left(t_{k}+\right.$ 1) $=\lim _{k} x_{s}\left(t_{k}\right)=x_{s}^{*}$, and hence

$$
\nabla C_{s}\left(x_{s}^{*}\right)\left(x_{s}-x_{s}^{*}\right) \geq 0
$$

for any feasible $x_{s}$. Summing over all $s$, we have for any feasible $x$

$$
\nabla C\left(x^{*}\right)\left(x-x^{*}\right) \geq 0
$$

which, since $C$ is convex, is necessary and sufficient for $x^{*}$ to be optimal.

\section{Proof of Theorem 3}

Since the cost functions $C_{l}$ are globally Lipschitz uniformly in $l$, the constant $A_{1}$ in (13) equals the Lipschitz constant $L$. For any $n$-tuple $z,\|z\|_{2} \leq \sqrt{n}\|z\|_{\infty}$, and hence the constant $A_{3}$ in (17) is $\pi$. Similarly, since $\|z\|_{1} \leq n\|z\|_{\infty}$, the constant $A_{4}$ in (18) is $\pi h$. By Lipschitz continuity, the constant $A_{5}$ in (19) is $A_{5}=A_{4} L=\pi h L$, the constant $A_{6}$ following is $A_{6}=A_{5} \lambda=$ $\pi h L \lambda$. Finally, since $\|z\|_{\infty} \leq\|z\|_{2}$, we have $A_{2}=A_{6}=$ $\pi h L \lambda$ in (20). Hence from (21) an upper bound for the stepsize $\gamma$ is

$$
\gamma<\frac{1}{A_{1}+A_{2}\left(2 t_{0}+1\right)}=\frac{1}{L\left(1+\pi h \lambda\left(2 t_{0}+1\right)\right)}
$$

